\title{
Transthoracic ultrasonic visualisation of coronary aneurysm, stenosis, and occlusion in Kawasaki disease
}

\author{
S Hiraishi, H Misawa, N Takeda, Y Horiguchi, N Fujino, N Ogawa, H Hirota
}

\begin{abstract}
Objective-To determine the sensitivity and specificity of our transthoracic echocardiographic technique using high frequency $(7.5 \mathrm{MHz})$ transducers for identification of the presence and type of coronary artery disease in patients with Kawasaki disease.

Design-The results of the prospective echocardiographic study in each of seven segments of the four major coronary arteries were compared with the selective coronary angiograms.

Setting-Kitasato University Hospital.

Subjects-60 patients with Kawasaki disease, ranging in age from 8.0 months to 22 years (median, 6.0 years).

Results-Adequate echocardiographic images were obtained in 397 (95\%) of 420 coronary segments. Coronary angiography showed the presence of coronary aneurysms in 87 segments and stenosis or occlusion in 28. The overall sensitivity and specificity of cross sectional echocardiography for correctly identifying coronary aneurysms were $95 \%$ and $99 \%$, respectively; for correctly identifying coronary stenosis or occlusion the values were $85 \%$ and $98 \%$ for the right coronary artery, and $80 \%$ and $97 \%$ for the left anterior descending coronary artery. Agreement on the presence or absence of coronary aneurysms and obstructive lesions on echocardiograms between the two observers was 1.0 and 0.98 , respectively.

Conclusions-Echocardiography may provide a non-invasive means of identifying the presence and type of coronary artery disease in patients with Kawasaki disease.

(Heart 2000;83:400-405)
\end{abstract}

Keywords: Kawasaki disease; coronary artery aneurysm; coronary artery stenosis; echocardiography

At present, the definitive way to identify coronary stenosis and distal lesions of the coronary arteries is cineangiography. ${ }^{1-3}$ However, it is sometimes difficult to monitor progressive change from aneurysm to stenotic lesion because angiography cannot be repeated very often. The ability to assess the patency of coronary arteries by non-invasive means would be an important advance. Kenny et al showed that high frequency epicardial echocardiography using a 7.5 or $10 \mathrm{MHz}$ transducer accurately and reproducibly measured coronary artery luminal diameter, detected qualitative changes in wall structure, and delineated stenotic or obliterated lesions. ${ }^{4}$ It is therefore possible that a transthoracic approach towards longitudinal imaging of the coronary arteries from multiple positions, together with technological improvements in image resolution, could allow recognition of coronary stenosis, occlusion, and distal lesions in coronary arteries. Our aim in this prospective study was to compare the results of non-invasive cross sectional echocardiography with findings obtained from coronary angiography in patients with Kawasaki disease.

\section{Methods}

\section{STUDY POPULATION}

During the 4.5 year study period from April 1994 to August 1998, 58 consecutive patients underwent routine coronary angiography for the evaluation of coronary sequelae in Kawasaki disease. Two patients with acute myocardial infarction who underwent emergency car- diac catheterisation for intracoronary thrombolytic treatment were also included in the study. The age range of the 60 patients was 8.0 months to 22 years (mean, 8.0 years; median, 6.0 years); 39 were male and 21 female. All patients were shown to have had coronary aneurysm formation in the acute stage of the disease, as documented by cross sectional echocardiography or coronary angiography. The interval between the onset of Kawasaki disease and the current study ranged from one month to 19.8 years (mean, 5.0 years; median, 3.0 years).

Our institutional committee on human research approved the study protocol, and informed consent was obtained from parents of all the children included in the study.

\section{ECHOCARDIOGRAPHY}

The 60 patients were studied prospectively with a commercially available system (Sonos 1500; Hewlett-Packard, Palo Alto, California, USA). Echocardiography was performed in all patients within three days of angiography. The coronary arteries were evaluated using a 7.5 $\mathrm{MHz}$ transducer with an axial resolution $<0.3$ $\mathrm{mm}$ at a focal zone of 2 to $6 \mathrm{~cm}$, which was automatically adjusted with the depth of imaging. The three major coronary arteries (right, left anterior descending, and left circumflex) and the left main coronary artery were arbitrarily divided into seven segments as follows: 


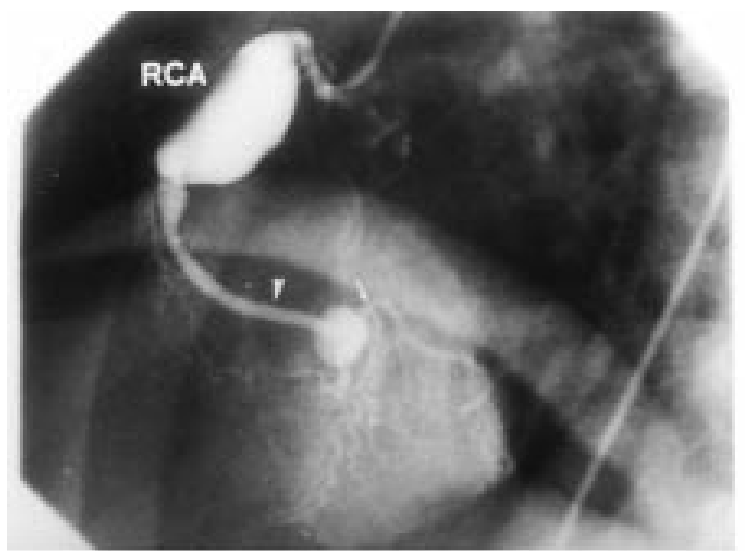

Figure 1 Upper panel: selective right coronary angiogram showing a fusiform aneurysm at the proximal to mid portions of the right coronary artery (segments 1 and 2) and a saccular aneurysm of the distal portion (segment 3) in a one year old boy. Lower panels: cross sectional echocardiograms from the same patient, showing good correspondence with the angiogram. The left and right images were obtained from modified subcostal views and the middle image from a modified apical view. AN, aneurysm; RCA, right coronary artery.

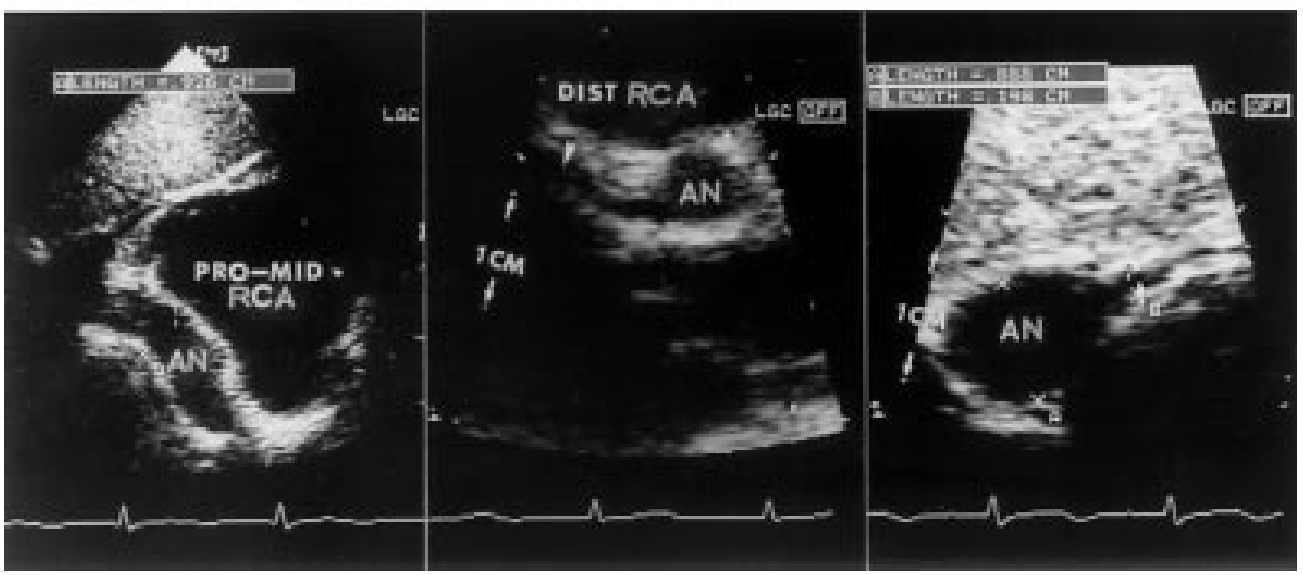

(1) Proximal, (2) mid, and (3) distal portions of the right coronary artery; (4) the left main coronary artery and the bifurcation; (5) proximal to mid left anterior descending artery; (6) the distal left anterior descending artery; and (7) the proximal to mid left circumflex artery. Each segment was visualised separately through different acoustic windows and the site of the coronary artery was magnified using the digital zoom feature of the scanning equipment (figs 1 and 2). We used the standard approach for the longitudinal imaging of segments 1,4 , and $5 .^{5-7}$ Other coronary regions such as segments $2,3,6$, and 7 were

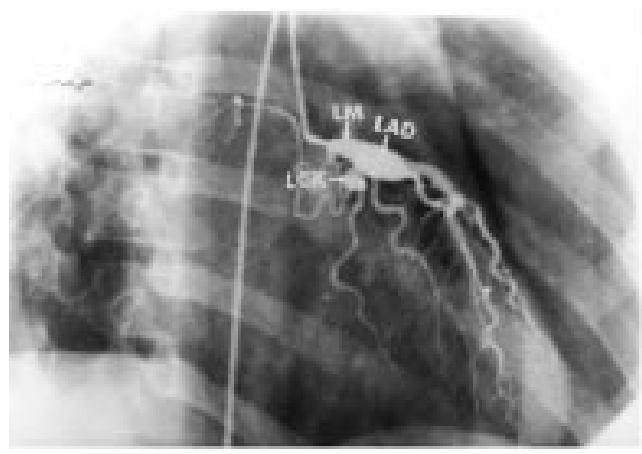

Figure 2 Left panel: selective left coronary angiogram showing a fusiform aneurysm at the left main and proximal left anterior descending coronary arteries in a six year old girl. Right panels: cross sectional echocardiograms from the same patient showing good correspondence with the angiogram. The four panels $(A, B, C, D)$ show images of segments $4,5,6$, and 7 , respectively. $A O$, aorta; $L A$, left atrium; $L A D$, left anterior descending coronary artery; $L M$, left main coronary artery; LCX, left circumflex artery; LV, left ventricle; $R V$, right ventricle. imaged using some modified techniques, as follows.

Segment 2-The patient was examined in the right lateral position. The transducer was located over the right parasternal border in the intercostal space 2, 3, or 4 and tilted leftwardinferiorly or sometimes leftward-superiorly with slight clockwise rotation. On this plane, two parallel linear echoes (a tubular structure) showing the mid portion of the right coronary artery were obtained along the tricuspid valve ring.

Segment 3-Visualisation of the distal portion of the right coronary artery began with the

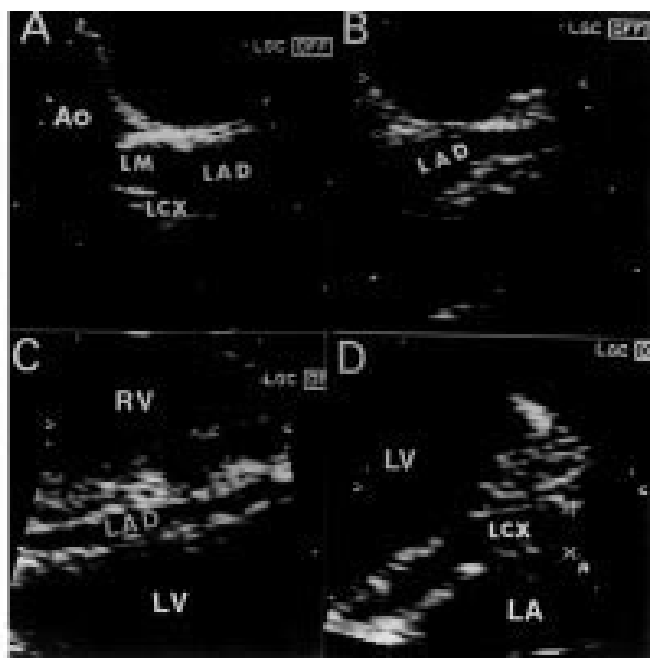


Table 1 Success rate of imaging seven segments of the major coronary arteries according to patient age

\begin{tabular}{|c|c|c|c|}
\hline \multirow[b]{2}{*}{ Segments } & \multicolumn{2}{|l|}{ Patient age } & \multirow[b]{2}{*}{$\begin{array}{l}\text { Overall } \\
(n=60)\end{array}$} \\
\hline & $\begin{array}{l}<10 \text { years } \\
(n=41)\end{array}$ & $\begin{array}{l}>10 \text { years } \\
(n=19)\end{array}$ & \\
\hline 1 & $41(100 \%)$ & $19(100 \%)$ & $60(100 \%)$ \\
\hline 2 & $38(93 \%)$ & $16(84 \%)$ & $54(90 \%)$ \\
\hline 3 & $39(95 \%)$ & $18(95 \%)$ & $57(95 \%)$ \\
\hline 4 & $41(100 \%)$ & $19(100 \%)$ & $60(100 \%)$ \\
\hline 5 & $39(95 \%)$ & $17(89 \%)$ & $56(93 \%)$ \\
\hline 6 & $39(95 \%)$ & $17(89 \%)$ & $56(93 \%)$ \\
\hline 7 & $39(95 \%)$ & $15(79 \%)$ & $54(90 \%)$ \\
\hline
\end{tabular}

standard apical four chamber view or slightly rightward of this position. The examination plane was then gradually directed rightwardinferiorly until the echoes from the tricuspid valve disappeared and the distal portion was detected as a tubular structure within the posterior atrioventricular groove. In addition, a subcostal approach was sometimes useful to image coronary segments 2 and 3 in infants and young children (fig 1).

Segment 6-After imaging the long axis view of the left ventricle from the left parasternal border in intercostal space 3 or 4 , the examination plane was gradually directed leftward-laterally until the echoes from the interventricular septum disappeared and the distal portion of the left anterior descending coronary artery was viewed as a tubular structure running along the anterior interventricular sulcus.

Segment 7-First, a long axis image of the left ventricle was imaged from the left parasternal border in the fourth or fifth intercostal space, or slightly leftward of this position. Then the ultrasound beam was gradually directed leftward-superiorly until the echoes from the mitral valve disappeared and the proximal $/ \mathrm{mid}$ portion of the left circumflex artery was identified as a tubular structure running along the mitral ring in its long axis.

ECHOCARDIOGRAPHIC EVALUATION

Real time images were stored on S-VHS videotape, permitting frame by frame analysis. For the purpose of this study, we chose adequate images in the evaluation of each segment rather than the landmark of the cardiac phase. Echocardiographic measurements of the coronary artery lumen were made with calipers from the video screen using the inner edge to inner edge method, and the values of three measurements were averaged. The reported data are the results from one observer $(\mathrm{SH})$.

ANGIOGRAPHY

The staff of the cardiac catheterisation laboratory, who were unaware of the echocardio-

Table 2 Sensitivity, specificity, and predictive value of cross sectional echocardiography in detecting coronary aneurysms

\begin{tabular}{lcccll}
\hline & \multirow{2}{*}{$\begin{array}{c}\text { No of segments } \\
\text { with aneurysm }\end{array}$} & $\begin{array}{c}\text { Sensitivity } \\
(\%)\end{array}$ & $\begin{array}{l}\text { Specificity } \\
(\%)\end{array}$ & & \multicolumn{2}{c}{ Predictive value } \\
\cline { 5 - 6 } Coronary artery & 39 & 95 & 99 & 0.96 & 0.99 \\
Right & 12 & 100 & 100 & 1.0 & 1.0 \\
Left main trunk & 96 & 99 & 0.96 & 0.96 \\
Left anterior descending & 27 & 89 & 100 & 1.0 & 1.0 \\
Left circumflex & 9 & 95 & 99 & 0.98 & 0.98 \\
Overall results & 87 & & & & Negative \\
\hline
\end{tabular}

graphic findings, interpreted the coronary angiographic images. Calibration for the measurements was accomplished by comparing their luminal boundary dimensions with those of the catheter used, according to the method of Vieweg et al. ${ }^{8}$

ANALYSIS OF DATA AND STATISTICS

Coronary lesions have been classified by the Research Committee on Kawasaki disease of the Japanese Ministry of Health and Welfare into two groups: dilated lesions and obstructive lesions. ${ }^{9}$ We considered that a localised stenosis was significant if it was greater than $50 \%$, relative to the nearest normal appearing portion of the coronary artery (either distal or proximal to the stenosis). When a localised narrowing was found among multiple coronary aneurysms at the proximal to mid portion of the major coronary arteries, coronary stenosis was defined in relation to normal values for age. ${ }^{10-12}$ The results of the echocardiographic study in each of the seven segments of the four major coronary arteries were compared with the selective coronary angiograms, and the sensitivity, specificity, and predictive value of echocardiography for identifying coronary aneurysm and stenosis/occlusion were determined. In these calculations, echocardiographic results for each segment that were thought to be uninterpretable by the observer (SH) were categorised as showing absence of coronary artery lesions in these segments. The presence or absence of coronary aneurysms and obstructive lesions on the echocardiograms were blindly assessed by a second observer (HM).

Interobserver variability of echocardiographic measurements of the diameters of the four major epicardial coronary arteries in our laboratory was determined in 20 randomly selected patients with Kawasaki disease. The mean interobserver difference was $6.3 \%$ for the proximal, mid, and distal regions of the right coronary artery (range $5.8 \%$ to $6.5 \%$ ); $5.8 \%$ for the left main coronary artery; $6.2 \%$ for the proximal, mid, and distal sites of left anterior descending coronary artery (range $6.0 \%$ to $6.4 \%$ ); and $7.2 \%$ for left circumflex coronary artery.

\section{Results}

Imaging and recording time of the echocardiographic examinations was from 15 to 30 minutes/patient. Image quality obtained by cross sectional echocardiography with the 7.5 $\mathrm{MHz}$ transducer was considered adequate in 397 (95\%) of 420 segments examined in the 60 patients (table 1). Angiography showed coronary aneurysms in 87 segments from 39 patients: segment 1, 22; segment 2, 15; segment 3, 2; segment 4, 29; segment 5, 8; segment 6,2 ; and segment 7,9 . The sensitivity, specificity, and predictive values of echocardiography for the correct identification of coronary aneurysms in each of the four major coronary arteries and the overall results are summarised in table 2 . Two aneurysms in the right coronary artery (segment 2 ), one in the 
Table 3 Sensitivity, specificity, and predictive value of cross sectional echocardiography in detecting obstructive coronary lesions

\begin{tabular}{|c|c|c|c|c|c|c|c|c|}
\hline \multirow[b]{2}{*}{ Coronary artery } & \multicolumn{4}{|c|}{ Obstructive lesions } & \multirow{2}{*}{$\begin{array}{l}\text { Sensitivity } \\
(\%)\end{array}$} & \multirow{2}{*}{$\begin{array}{l}\text { Specificity } \\
(\%)\end{array}$} & \multicolumn{2}{|c|}{ Predictive value } \\
\hline & $n$ & Local & Seg & Occlu & & & Positive & Negative \\
\hline Right coronary & 13 & 6 & 5 & 2 & 85 & 98 & 0.79 & 0.99 \\
\hline Left anterior descending & 15 & 7 & 3 & 5 & 80 & 97 & 0.80 & 0.97 \\
\hline
\end{tabular}

Local, localised stenosis; Occlu, occlusion; Seg, segmental stenosis.

left anterior descending coronary artery (segment 5), and one in the left circumflex coronary artery were not defined by echocardiography.

Angiography also showed 28 coronary obstructive lesions in 18 patients: 15 in the left anterior descending coronary artery (segment 4,7 ; segment 5,7 ; segment 6,1 ) and 13 in the right coronary artery (segment 1,7 ; segment 2 , $6)$. In these patients, eight had single vessel and 10 two vessel stenosis or occlusion. There were no obstructive lesions in the left main and circumflex arteries. The sensitivity, specificity, and predictive values of echocardiography for correctly identifying coronary stenosis and occlusion in the left anterior descending and right coronary arteries are shown in table 3. Two examples of a series of echocardiographic views in patients with segmental stenosis or thrombotic obstruction are shown in figs 3 and 4 , with corresponding coronary angiograms. Of the five segments erroneously classified by echocardiography as not having an obstructive lesion (false negative results), two had occlusion within the lumens of aneurysms, with severe calcification of the walls, in the right coronary artery and the left anterior descending coronary artery. The other three vessels had localised segmental stenoses in the right coronary artery (segment 2) and localised ste- nosis in the left anterior descending coronary artery (segment 4 ). Six segments (segment 1 , 1 ; segment 2 , 2; segment 4,2 ; segment 5,1 ) were interpreted as being abnormal on the echocardiogram but angiography showed slight narrowing (20-40\%) in three and normal coronary arteries in three (false positive result).

OBSERVER VARIABILITY

Agreement on the presence or absence of coronary aneurysms and obstructive lesions on echocardiograms between the two observers was 1.0 and 0.98 , respectively. One vessel with segmental stenosis on angiography was judged to be normal by one observer. Other interobserver discrepancies were caused by problems in selection and evaluation of the coronary images in four segments and by a small difference in the value measured at the stenotic site in two segments. These differences in judgment between the independent observers were resolved by consensus after re-evaluation of the images.

\section{Discussion}

Our success in imaging appears to be related to several features of the imaging technique. First, we considered that the positive identification of significant lesions requires visualisation of both proximal and distal portions of arteries containing suspected lesions. Accordingly, the coronary artery system was divided into seven segments, where each was separately visualised parallel to its long axis using modified acoustic windows. The validity of our echocardiographic technique was confirmed by good agreement with coronary angiography. Second, we found that the $7.5 \mathrm{MHz}$ transducer produced qualitatively better images for the

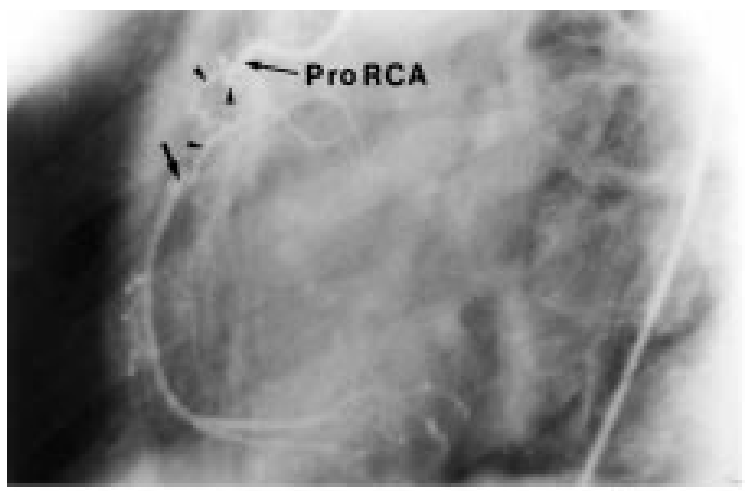

Figure 3 Upper panel: selective right coronary angiogram shows segmental stenosis (a bridging lesion; arrowheads) in the proximal right coronary artery (Pro RCA) in a 20 year old man. Lower panels: echocardiograms from the same patient; left and middle panels: proximal right coronary artery (Pro RCA) showed abrupt separation into two smaller vessels; right panel: another abnormal vessel (arrowhead) was viewed by more leftward-inferior angling of the transducer than the plane recorded for the middle panel. These were judged to be bridging vessels connecting the site of an occlusion.

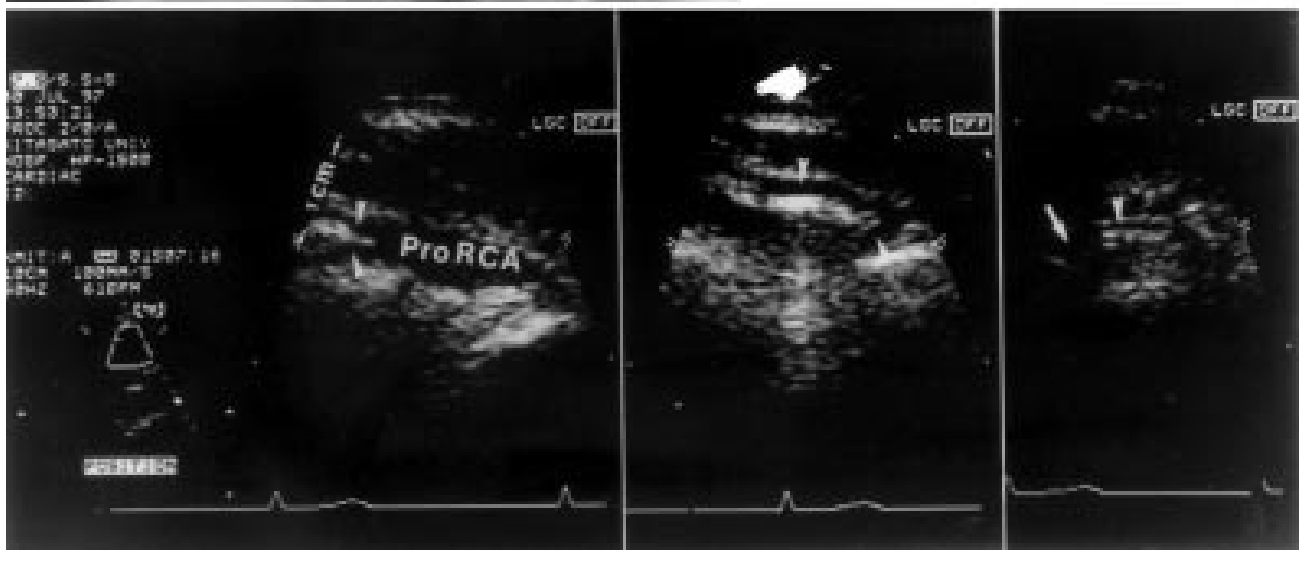



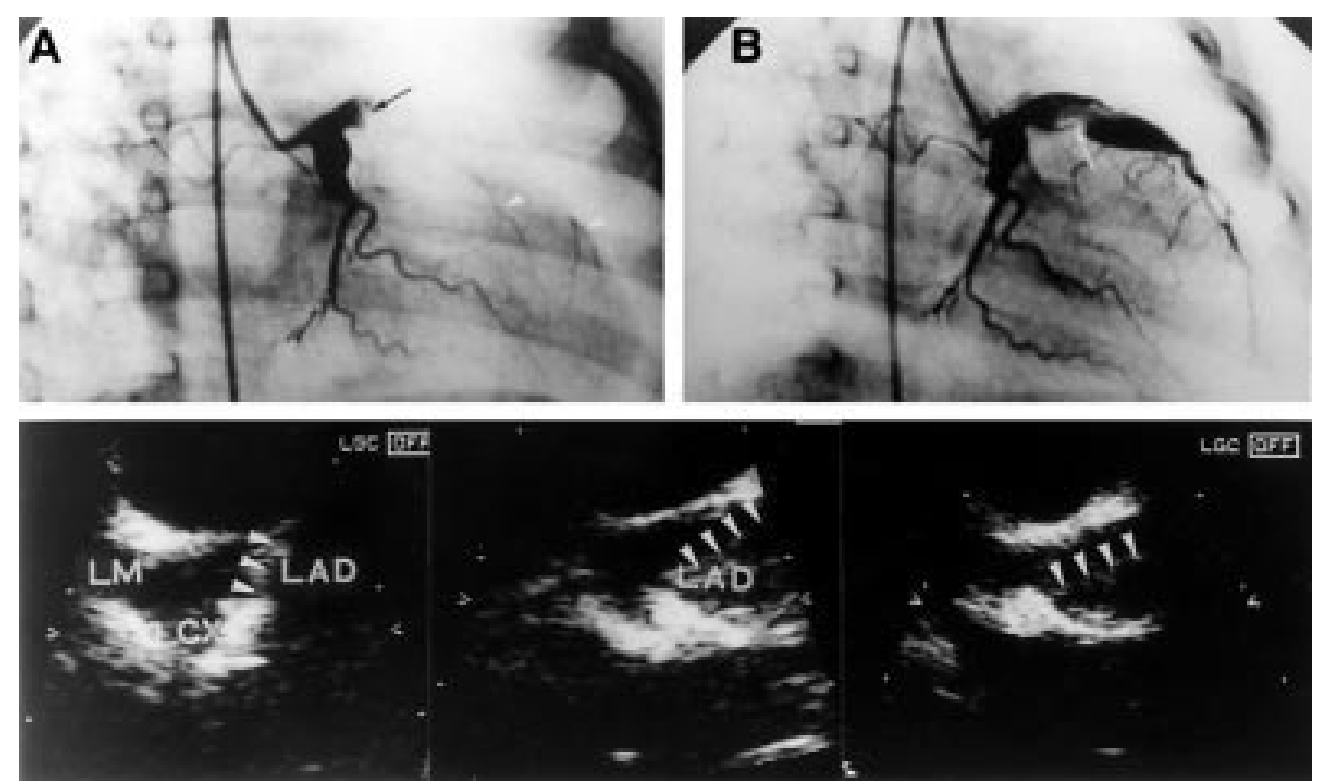

Figure 4 Upper panels: selective left coronary angiograms before and after intracoronary thrombolytic treatment in a one year old boy, showing partial thrombolysis within the aneurysm of the proximal left anterior descending coronary artery (LAD). Lower panels: serial cross sectional echocardiograms from the same patient. The left and middle panels show images before and one hour after intracoronary thrombolytic treatment. The right panel is the echocardiogram done one day after thrombolytic treatment.

identification of coronary artery lesions, especially coronary stenosis and occlusion. In addition, the digital zoom feature on the scanning equipment enabled us to measure the coronary artery lumen diameter accurately. Presti et al reported values for sensitivity and specificity for detecting stenotic lesions in the proximal left anterior descending coronary artery by transthoracic echocardiography of $98 \%$ and $67 \%$, respectively. ${ }^{13}$ In that study, examinations were performed in adult patients, in whom a 7.5 $\mathrm{MHz}$ transducer may not provide adequate power for imaging. More recently, two studies have shown that transthoracic high frequency echocardiography can allow visualisation of the distal left anterior descending coronary artery, but identified only one patient with stenosis in each study. ${ }^{14}{ }^{15}$ To our knowledge, ours is the first study to evaluate the sensitivity, specificity, and predictive value of cross sectional echocardiography for identifying the presence and type of coronary artery disease and to compare it with angiography in a relatively large number of patients with Kawasaki disease.

CLINICAL IMPLICATIONS

A previous study using postmortem comparisons of echocardiography and angiography showed that the echocardiographic technique may not reveal the lumen of the aneurysm when there is occlusion of the coronary artery lumen by fresh or organised thrombi, or when there is marked thickening of the vessel wall. ${ }^{16}$ In the present study, however, it was possible to detect fresh thrombus within the lumen of a large aneurysm, and our results were consistent with other reports. ${ }^{17}{ }^{18}$ Additionally, our technique detected localised and segmental stenosis with a braid-like or bridging lesion, which was considered to represent recanalisation of a thrombotic occlusion. ${ }^{19}$
In patients with complete occlusion, there were uneven high intensity echoes within a dilated coronary artery, or an abrupt cessation of the luminal echo signal. Recently, transoesophageal echocardiography, performed using high frequency transducers, has been shown to detect proximal coronary artery stenosis. ${ }^{20}$ The clinical value of this method is limited, however, to proximal coronary disease, and such an approach would be invasive, especially in children. Thus the present study suggests that our segmental approach to imaging coronary arteries could be valuable in identifying the presence and type of coronary artery disease in patients with Kawasaki disease.

\section{LIMITATIONS}

Although our echocardiographic technique resulted in acceptable coronary artery images in a large proportion of the patients, views were sometimes limited in imaging the mid portion of the right coronary artery and the left circumflex artery in older patients. The other problem in the echocardiographic assessment of obstructive coronary artery lesions was that in some patients with highly echogenic lesions, such as calcification in the aneurysm wall, there was acoustic shadowing, and resolution of the vessel lumen was limited (false negative results). On the other hand, the coronary arteries moved in and out of the imaging plane used in this study in concert with cardiac motion. Interpretation of inadequate stop frames caused false positive results. Thus the examiner should judge carefully whether the aneurysmal and obstructive areas of arterial segments visualised represent real time images, in order to lessen false positive interpretation. 
CONCLUSIONS

Our preliminary data suggest that in its current state of development, echocardiography may be helpful as a screening tool during follow up, limiting the need to repeat catheterisations.

We thank Mr Yoshiyuki Makita, Mrs Keiko Fukuoka, and Miss Saori Tanaka for technical assistance and Professor Nobuo Matsuura for useful comments.

1 Pahl E, Ettedgui J, Neches WH, et al. The value of angiography in the follow-up of coronary involvement in mucocutaneous lymph node syndrome (Kawasaki disease). f Am Coll Cardiol 1989;14:1318-25.

2 Suzuki A, Kamiya T, Ono Y, et al. Follow-up study of coronary artery lesions due to Kawasaki disease by serial selective coronary arteriography in 200 patients. Heart Vessels 1987 3:159-65.

3 Capannari TE, Daniels SR, Meyer RA, et al. Sensitivity, specificity and predictive value of two-dimensional echocardiography in detecting coronary artery aneurysms in patients with Kawasaki disease. $\mathcal{F} \mathrm{Am}$ Coll Cardiol 1986;7:355-60.

4 Kenny A, Fuller CA, Cary NRB, et al. Histopathological validation of high frequency epicardial echocardiography of the coronary arteries in vitro. Br Heart $\mathcal{f} 1991 ; 65$ : of the cor 31 .

5 Weyman AE, Feigenbaum H, Dillon JC, et al. Noninvasive visualization of the left main coronary artery by cross sectional echocardiography. Circulation 1976;54:169-74.

6 Saito A, Ueda K, Nakano H. Two-dimensional echocardiographic visualization of the peripheral right coronary artery in patients with mucocutaneous lymph node syndrome. $f$ Cardiogr 1982;12:401-13.

7 Douglas PS, Fiolkoski J, Berko B, et al. Echocardiographic visualization of coronary artery anatomy in the adult. $\mathcal{f} A m$ Coll Cardiol 1988;11:565-71.

8 Vieweg WVR, Alpert JS, Hagan CAD. Caliber and distribution of normal coronary arterial anatomy. Cathet Cardiovasc Diagn 1976;2:269-80. $9 \begin{aligned} & \text { Kamiya T, Kawasaki T, Okuni M, et al. Diagnostic criteria of } \\ & \text { cardiovascular lesions in Kawasaki disease. Research Com- }\end{aligned}$ cardiovascular lesions in Kawasaki disease. Research Com-
mittee on Kawasaki Disease Ministry of Health and Wittee on Kawasaki Disease Ministry of Health and 1984:1-10.

10 Arjunan K, Daniels SR, Meyer RA, et al. Coronary artery caliber in normal children and patients with Kawasaki disease but without aneurysms: an echocardiographic and angiographic study. F Am Coll Cardiol 1986;8:1119-24.

11 Nakano H, Ueda K, Saito A, et al. Repeated quantitative angiograms in coronary arterial aneurysm in Kawasaki disease. Am F Cardiol 1985;56:845-51.

12 Oberhoffer R, Lang D, Feilen K. The diameter of coronary arteries in infants and child

13 Presti CF, Feigenbaum H, Armstrong WF, et al. Digital two-dimensional echocardiographic imaging of the proximal left anterior descending coronary artery. Am $\mathcal{F}$ Cardiol 1987;60:1254-9.

14 Ross JJ, Mintz GS, Chandrasekaran K. Transthoracic two-dimensional high frequency $(7.5 \mathrm{MHz})$ ultrasonic visualization of the distal left anterior descending coronary artery. 7 Am Coll Cardiol 1990;15:373-7.

15 Kenny A, Shapiro LM. Transthoracic high-frequency two-dimensional echocardiography, Doppler and color flow mapping to determine anatomy and blood flow patterns in the distal left anterior descending coronary artery. Am F Cardiol 1992;60:1265-8.

16 Fujiwara T, Fujiwara H, Ueda T, et al. Comparison of macroscopic, postmortem, angiographic and two-dimensional echocardiographic findings of coronary aneurysms in children with Kawasaki disease. Am 7 Cardiol 1986;57:761-4.

17 Yanagisawa M, Yano S, Shiraishi H, et al. Coronary aneurysms in Kawasaki disease: follow-up observation by twoin Kawasaki disease: follow-up observation by two-
dimensional echocardiography. Pediatr Cardiol 1985;6:11-16.

18 Minich LL, Tani LY, Pagotto LT, et al. Usefulness of echocardiography for detection of coronary artery thrombi in patients with Kawasaki disease. Am f Cardiol 1998;82: patients

19 Naoe S, Shibuya K, Takahashi K, et al. Pathological observations concerning the cardiovascular lesions in Kawasaki disease. Cardiol Young 1991;1:212-20.

20 Samdarshi TE, Nanda NC, Gatewood RP, et al. Usefulness and limitations of transesophageal echocardiography in the assessment of proximal coronary artery stenosis. $7 \mathrm{Am}$ Coll Cardiol 1992;19:572-80. 